\title{
Load Based Cell Selection Algorithm for Tetra based Professional Mobile Radio
}

\author{
Azad Karataş, Izmir Institute of Technology, Berna Özbek, Izmir Institute of Technology, \\ İlker Sönmez, Aselsan A.Ş., Sidıka Bengür, Aselsan A.Ş.
}

\begin{abstract}
This paper proposes a cell selection algorithm for Tetra based professional mobile radio systems where the users select the base station according to the proposed utility value. This utility value is determined by considering both cell load and received signal strength indicator. The main aim of the proposed method is to distribute the users in a balanced way among base stations. Based on performance evaluations, we illustrate that the proposed cell selection algorithm gives better results than Tetra based cell selection method in a manner of distribution of the users over all BSs while reducing waiting time for establishing connection.
\end{abstract}

Keywords - cell selection, PMR, Tetra, wireless communication.

\section{INTRODUCTION}

$\mathrm{P}$ rofessional Mobile Radio (PMR) is widely used by emergency services. PMR systems should keep working even in case of disaster and even when the conventional public networks are out of order. PMR systems provide facilities for group call, user groups and push-to-talk. Their call setup time is less than cellular systems which plays critical role in emergency situations [1]. It refers to the two-way radio communication system. Tetra is a technical platform providing integrated voice and data services and modern standard for digital PMR. Its first version was published in 1995 and it is an ETSI standard. It is designed for emergency services, government agencies, military and transport services. There are many advantages that provide Tetra is an ideal choice for many radio communications requirements such as security, easy to use and fast call set-up times. Tetra mobile users have an ability to communicate in both way, direct-mode operation or trunked-mode operation. Trunk radio systems work hardly in emergency cases because of saturation of the access channels of the base station (BS) that causes large delays and sometimes the communications becomes impossible. Even in this

Azad Karatas is with the Department of Electrical and Electronics Engineering, Izmir Institute of Technology, Urla, İzmir, 35430, Turkey, (phone: 90-232-7506527; e-mail: azadkaratas@iyte.edu.tr)

Berna Özbek is with the Department of Electrical and Electronics Engineering, Izmir Institute of Technology, Urla, İzmir, 35430, Turkey, (e-mail: bernaozbek@iyte.edu.tr)

İlker Sönmez, Aselsan A.Ş., Yenimahalle, Ankara, 06172, Turkey, (email: isonmez@aselsan.com.tr)

Sidıka Bengür, Aselsan A.Ş., Yenimahalle, Ankara, 06172, Turkey, (e-mail: sbengur@aselsan.com.tr) overloaded situations, a dedicated radio system should overcome this problem to provide successful communication.

Cell selection is the process of deciding the BS to provide services to the mobile users. In [2], a novel cell selection algorithm has been performed by utilizing a mathematical model of proportional fair scheduling algorithm to assist a new user to select the best serving cell by achieving maximum achievable data rate. In [3], in order to achieve proportional fairness for all users, a cell selection has been formulated into a network-wide utility maximization problem. In [4], a cell selection algorithm has been performed for mobile networks with backhaul capacity constraints. This model analyzes the possibility to exploit load balancing among BSs to improve backhaul capacity utilization. In [5], an intelligent cell selection has been handled to satisfy user's requirements as well as system requirements for inter system handover in heterogeneous networks. This procedure needs the aggregation functions based on fuzzy set theory. In [6], a preference value-based cell selection has been performed for heterogeneous wireless access environment. This cell selection contains three stages including candidate cells selection, preference value calculation, and target cell determination. In [7], an all-or-nothing demand maximization algorithm has been performed to satisfy each mobile user's requirements by finding a maximumprofit subset of clients. In [8], we have examined Tetra based cell selection algorithm for Tetra trunk systems by considering practical constraints.

Except the work presented in [8], all existing works have been developed for mainly cellular wireless networks and cannot be directly applied to PMR systems. In this paper, we propose a cell selection algorithm that takes into account both cell load and received signal strength indicator (RSSI) to reduce transmission delay. The rest of the paper is organized as follows. Section II describes the system model. Section III introduces the proposed cell selection algorithm. Section IV is dedicated to simulation parameters and performance results. Finally, section V gives the conclusions.

\section{SYSTEM MODEL}

Tetra uses Time Division Multiple Access (TDMA) technique with four user channels on one radio carrier with $25 \mathrm{kHz}$ spacing between carriers by employing $\pi / 4$ DQPSK modulation scheme [9]. BS $u$ has $M_{u}$ timeslots which is defined as follows: 


$$
M_{u}=4 \frac{B}{\Delta \mathrm{f}}
$$

where $B$ represents the bandwidth per cell and $\Delta f$ is channel spacing. One of the timeslots can be used for control channel.

For Tetra Trunk systems, the path loss parameter C1 [9] is used for cell selection given as:

$$
\begin{aligned}
& C 1_{u, k}=R S S I_{u, k}-R x_{-} \text {Lev_Access_Min- } \\
& \operatorname{Max}\left(0, M S_{-} T x P w r_{-} \text {Max_Cell }{ }_{u}-P_{\text {MSMAX }}\right)
\end{aligned}
$$

where $\operatorname{RSSI}_{u, k}$ is the RSSI value of user $k$ belonging to BS $u$, Rx_Lev_Access_Min is the minimum acceptable received power at mobile user, $M S_{-} T x P w r_{-} M_{-}$Cell $_{u}$ denotes the maximum allowed transmit power for BS $u$ and $P_{M S M A X}$ is the maximum transmit power in $\pi / 4$ DQPSK modulation.

The RSSI belonging to $u$ th BS for user $k$ is determined by,

$$
R S S I_{u, k}=E I R P_{u}-P L_{u, k}-B u L-S h_{u, k}-B L+G_{r}-C L
$$

where $C L$ is receiver cable loss, $G_{r}$ is receiver antenna gain, $P L_{u, k}$ is path loss between user $k$ and $\mathrm{BS} u, B L$ is body loss, $S h_{u, k}$ is shadowing effect modelled by $\log$ normal distribution, $B u L$ is building loss when the user is inside, $P L_{u, k}$ is the path loss modelled as Hata model [10] Effective isotropic radiated power (EIRP) for $\mathrm{BS} \mathrm{u}$ is given by,

$$
E I R P_{u}=P_{u}^{t}+G_{t}-C L_{t}
$$

where $P_{u}^{t}$ is transmit power, $G_{t}$ is transmitter antenna gain and $C L_{t}$ is transmitter antenna cable loss.

For Tetra based cell selection algorithm, each mobile user adds a BS with $\mathrm{C} 1$ parameter that is higher than zero to its candidate set and then it selects the BS by,

$$
u^{*}=\arg \max _{1 \leq u \leq N C} C 1_{u, k}, \forall k
$$

where $N C$ is the total number of BSs in the candidate set.

Besides Tetra based cell selection, it is possible to apply signal-to-noise ratio (SINR) based cell selection as:

$$
u^{\prime \prime}=\arg \max _{1 \leq u \leq N B} \operatorname{SINR}_{u, k}, \forall k
$$

where $N B$ is total number of BSs in the Tetra trunk system in the considered area and $S I N R_{u, k}$ is the SINR of user $k$ belonging to $\mathrm{BS} u$ and it is determined by,

$$
\operatorname{SINR}_{u, k}=\frac{P_{u}^{r}}{I N T^{r}+N_{0} B_{t}}
$$

with $P_{u}^{r}$ is the received power from BS $u, I N T$ is the interference power caused by the other cells having the same frequency, $N_{0}$ is the noise spectral density, $B_{t}$ is the transmission bandwidth. Interference power can be determined by assuming that the cell planning is known at each user.

\section{Proposed Cell Selection Algorithm}

The cell load needs to be considered while assigning the users to the BSs. The unbalanced distribution of users among cells may cause higher waiting time to communicate because of the limited number of available channels. Therefore, we propose a cell selection algorithm which considers both cell load and RSSI. The procedures of proposed algorithm is given in the following:

\section{Constructing the set based on receiver sensitivity:}

Each user $k$ measures the received signal strength from all BSs. The set is constructed by the BSs whose RSSI value exceeds a given signal strength threshold, $R S S I_{t h}$ :

$$
P_{k}=\left\{u \in N B: R S S I_{u, k} \geq R S S I_{t h}\right\}
$$

\section{Utility Value Calculation:}

The user calculates the utility value belonging to each BS in the set $P_{k}$. The utility value is calculated by taking into account both RSSI value and cell load parameter of the BSs.

$$
U_{u, k}=w f\left(R S S I_{u, k}\right)+(1-w) g\left(U C L_{u}\right), \forall u, \forall k
$$

where $w$ is the weight of RSSI value and (1-w) is the weight of mapped cell loading. The function $\mathrm{f}($.) represents the transformation of RSSI values and the function $\mathrm{g}($.$) transforms the unmapped cell load (UCL) to$ mapped cell load.

The UCL of BS $u$ is calculated as,

$$
U C L_{u}=(1-c) \frac{A_{u}}{M_{u}}+c \frac{I_{u}}{K_{u}}
$$

where $A_{u}$ and $I_{u}$ are the number of active and inactive users attaching to $u$ th base station, respectively. Active users are attached to a cell and communicating whereas inactive users are only attached but not communicating. $c$ represents the importance of contribution of inactive users while determining the cell load. $M_{u}$ and $K_{u}$ determines the number of active and inactive users per BS in the considered system, respectively. $K_{u}$ is calculated as:

$$
K_{u}=\frac{N U}{N B}-M_{u}
$$

where $N U$ is total number of users for the considered area.

\section{Attaching the BS:}

In the proposed cell selection algorithm, each user is attached to BS by,

$$
u^{*}=\arg \max _{u \in P_{k}} U_{u, k}, \forall k
$$

The aim of the proposed cell selection algorithm is to attach the users to BS having the maximum utility value to balance the users among to BSs.

The flowchart of the proposed algorithm is shown in Fig. 1. 


\section{Performance EVAluations}

While providing performance evaluations, we consider only voice users which need only one channel. Indoor users have extra building loss. In the BSs, there are more than one antennas to achieve uplink diversity where the signal having highest power is selected.

The number of active users varies depending on the traffic load of the system. Only high traffic load is considered in simulations where the percentage of active users is $50 \%$. The proposed algorithm is evaluated for using two different weights.

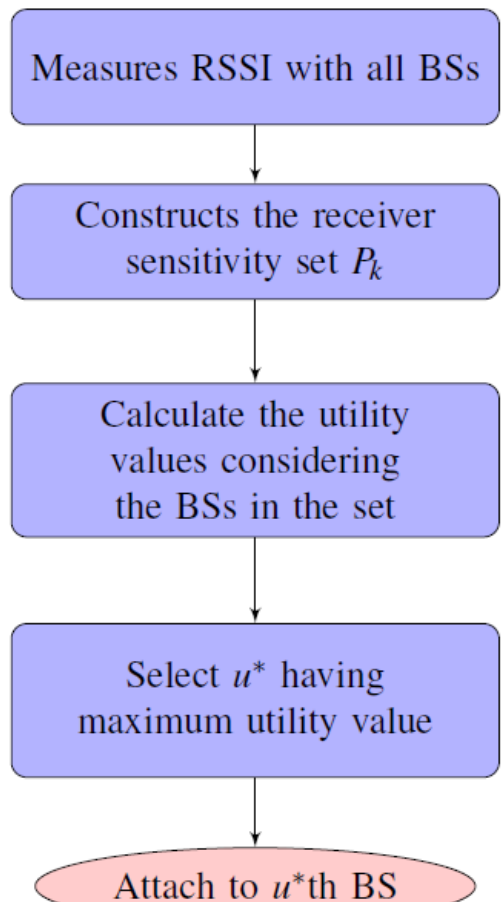

Fig. 1. Flowchart of proposed cell selection algorithm

While calculating utility value, transformation of RSSI values is done in $f$ function and mapping of cell load is calculated in $\mathrm{g}$ function. In $f$ function, the measured RSSI values are sorted and the function returns a mapped value corresponding RSSI according to Table 1 in mobile user. In $g$ function, the cell load is mapped as given in Table 2 and then it is broadcasting by BS by using 2 bit. The simulation parameters are listed in Table 3.

TABLE 1: MAPPING FUNCTION OF RSSI VALUES IN $f$ FUNCTION

\begin{tabular}{|c|c|c|c|}
\hline Sorted RSSI & $\boldsymbol{f}()$. & $\begin{array}{c}\text { Sorted } \\
\text { RSSI }\end{array}$ & $\boldsymbol{f}()$. \\
\hline 1 & 1 & 8 & 0.5 \\
\hline 2 & 0.928 & 9 & 0.429 \\
\hline 3 & 0.857 & 10 & 0.357 \\
\hline 4 & 0.786 & 11 & 0.286 \\
\hline 5 & 0.714 & 12 & 0.214 \\
\hline 6 & 0.643 & 13 & 0.143 \\
\hline 7 & 0.571 & 14 & 0.071 \\
\hline
\end{tabular}

The considered urban area cell model is shown in Fig. 2. In this area, there are $\mathrm{NB}=14$ base stations and $\mathrm{NU}=600$ users which are uniformly distributed in the coverage area. Each base station has 24 channels. The BSs with same color have same carrier frequency and frequency reuse factor is chosen as 1:7

Table 2: Mapping of Cell Load Values in $g$ Function

\begin{tabular}{|c|c|c|}
\hline $\boldsymbol{U C L}$ & Index & $\boldsymbol{g}()$. \\
\hline $0-0.5$ & 1 & 1 \\
\hline $0.5-0.8$ & 2 & 0.66 \\
\hline $0.8-1$ & 3 & 0.33 \\
\hline
\end{tabular}

TABLE 3: Simulation PARAMETERS

\begin{tabular}{|c|c|}
\hline PARAMETERS & Tetra \\
\hline Transmit Power & $44 \mathrm{dBm}$ \\
\hline Transmission Bandwidth & $20 \mathrm{kHz}$ \\
\hline Channel Spacing $(\Delta f)$ & $25 \mathrm{kHz}$ \\
\hline Noise Spectral Density & $-174 \mathrm{dBm} / \mathrm{Hz}$ \\
\hline Receiver Sensitivity & $-115 \mathrm{dBm}$ \\
\hline TX Antenna Gain & $8 \mathrm{~dB}$ \\
\hline TX Cable Loss & $6 \mathrm{~dB}$ \\
\hline RX Antenna Gain & $-2 \mathrm{~dB}$ \\
\hline RX Cable Loss & $0 \mathrm{~dB}$ \\
\hline BS Antenna Height & $30 \mathrm{~m}$ \\
\hline MS Antenna Height & $1.5 \mathrm{~m}$ \\
\hline Building Loss & $16.5 \mathrm{~dB}$ \\
\hline Weight of RSSI $(\mathrm{w})$ & $0.3,0.5$ \\
\hline Parameter c & 0.1 \\
\hline Percentage of Indoor Users & $30 \%$ \\
\hline
\end{tabular}

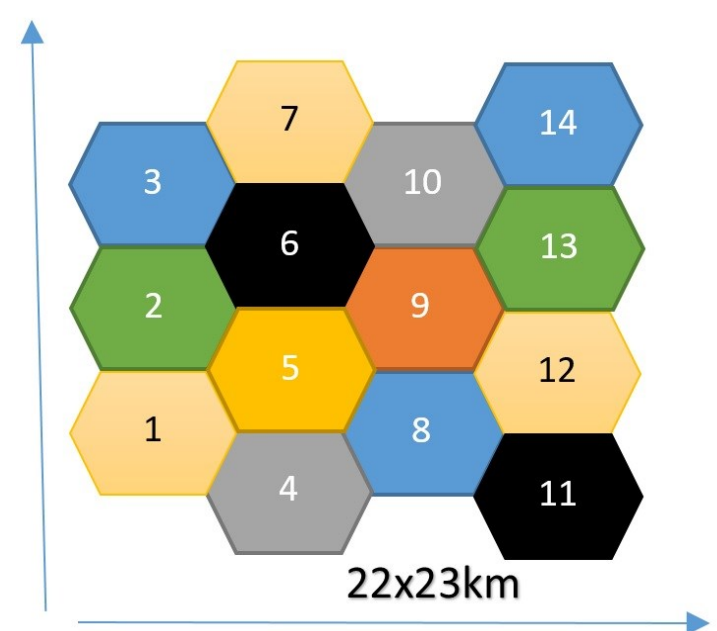

Fig. 2. $22 \mathrm{~km}$ x 23km Urban Model

The performance of cell selection algorithms are investigated by considering at Load Fairness Index (LFI), which is calculated as:

$$
L F I=\frac{N U^{2}}{N B \sum_{u=1}^{N B} S_{u}^{2}}
$$

where $S_{u}$ is the total number of active and inactive users in BS $u$.

The other metrics considered to compare performance 
results are the average user counter, which is the total number of requests to be attached to a BS and the number of waiting user which is the number of unattached active users and the BER performance of active users.

Fig. 3 shows the distribution of users among BSs. It is shown the fairness is satisfied by SINR and Tetra based algorithms compared to the proposed algorithm, which is undesirable since it increases the waiting time for connection. In Table 4, it is given that the average user counter and the number of waiting users are much lower for the proposed algorithm which leads to design practical systems while improving the service quality in Tetra based systems. The load fairness index is almost 1 for the proposed algorithm, which is desirable and also demonstrates the effect of variation of distributed users. Besides, the required outage probability for voice transmission, which must be lower than $5 \%$, is also satisfied for the proposed cell selection algorithm as seen in Table 5.

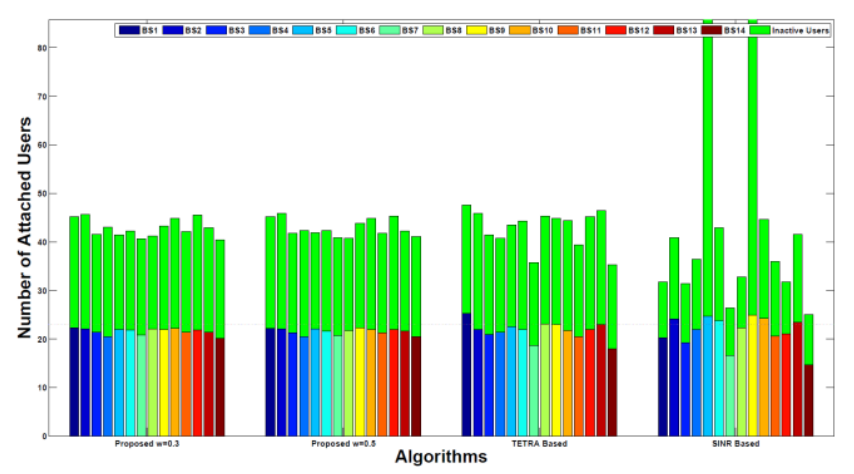

Fig. 3. The distribution of the users among BS for different algorithms

TABLE 4: THE RESULTS ON DEFINED METRICS FOR URBAN AREA

\begin{tabular}{|c|c|c|c|}
\hline $\begin{array}{c}\text { Algorithms } \\
\text { \& } \\
\text { Results }\end{array}$ & $\begin{array}{c}\text { Load } \\
\text { Fairness } \\
\text { Index }\end{array}$ & $\begin{array}{c}\text { Average } \\
\text { User } \\
\text { Counter }\end{array}$ & $\begin{array}{c}\text { Number of } \\
\text { Waiting } \\
\text { Users }\end{array}$ \\
\hline Tetra Based & 0.977 & 1.1037 & 7 \\
\hline SINR Based & 0.811 & 1.6835 & 7.34 \\
\hline $\begin{array}{c}\text { Proposed Alg. } \\
\text { w=0.3 }\end{array}$ & 0.987 & 1.0158 & 2.8 \\
\hline $\begin{array}{c}\text { Proposed Alg. } \\
\text { w }=0.5\end{array}$ & 0.987 & 1.0177 & 2.9 \\
\hline
\end{tabular}

TABLE 5: BER PERFORMANCE

\begin{tabular}{|c|c|}
\hline Algorithms & $\begin{array}{c}\text { Outage } \\
\text { Probability }\end{array}$ \\
\hline Tetra Based & 0.0014 \\
\hline SINR Based & 0.0011 \\
\hline $\begin{array}{c}\text { Proposed Alg. } \\
\text { w }=0.3\end{array}$ & 0.0111 \\
\hline $\begin{array}{c}\text { Proposed Alg. } \\
\text { w }=0.5\end{array}$ & 0.0066 \\
\hline
\end{tabular}

\section{CONCLUSION}

We have proposed a cell selection method which is suitable for practical applications for Tetra trunk systems. The main target of the proposed algorithm is to increase the load fairness index to distribute the users among the cells fairly. In the proposed algorithm, the users are attached to BS considering the utility values calculated based on RSSI value and cell load. Therefore, each user selects the BS that has the highest utility value. We have obtained the performance results in an urban area with high traffic condition since the waiting time to connect the BS is very critical for these crowded areas. According the performance results, we have shown that proposed cell selection algorithm gives the best performance in a manner of distribution of active and inactive users over all BSs while satisfying the required outage probability to establish reliable transmission.

\section{ACKNOWLEDGMENT}

This work has been supported by Republic Ministry of Science, Industry and Technology under SAN-TEZ 0686.STZ.2014 Programme.

\section{REFERENCES}

[1] ETSI, "Private Mobile Radio". 1998.

[2] M. A. AboulHassan, E. A. Sourour and S. E. Shaaban, "Novel cell selection algorithm for improving average user's effective data rate in LTE HetNets," 2014 IEEE Symposium on Computers and Communications (ISCC), Funchal, 2014, pp. 1-6.

[3] J. Wang; J. Liu; D. Wang; J. Pang; G. Shen, "Optimized Fairness Cell Selection for 3GPP LTE-A Macro-Pico HetNets", Vehicular Technology Conference (VTC Fall), IEEE, 2011, pp. 1-5.

[4] J. J. Olmos, R. Ferrus and H. Galeana-Zapien, "Analytical Modeling and Performance Evaluation of Cell Selection Algorithms for Mobile Networks with Backhaul Capacity Constraints," in IEEE Transactions on Wireless Communications, vol. 12, no. 12, pp. 6011-6023, December 2013.

[5] J. C. Lee and S.-M. Yoo, "Intelligent cell selection satisfying user requirements for inter-system handover in heterogeneous networks," Comput. Commun., vol. 35, pp. 2106-2114, 2012.

[6] C. J. Chang, C. Y. Hsieh and Y. H. Chen, "A Preference ValueBased Cell Selection Scheme in Heterogeneous Wireless Networks," 2010 IEEE Wireless Communication and Networking Conference, Sydney, NSW, 2010, pp. 1-6.

[7] M. Amutha Surya, Dr. Sangeetha SenthilKumar, "An Efficient Cell Selection in Cellular Network With High Scalability", International Journal of Engineering Research and Technology (IJERT) Vol. 3 Issue 1, January 2014.

[8] A. Karataş, B. Özbek, E. D. Bardak and İ. Sönmez, "Cell selection algorithm performance for Tetra trunk," 2016 24th Signal Processing and Communication Application Conference (SIU), Turkey, 2016, pp. 373-376.

[9] ETSI, "TS 100 392-2 V3.6.1", May, 2013.

[10] ETSI Technical Report143 030 V9.0.0 (2010-02), “Digital cellular telecommunications system (Phase 2+); Radio network planning aspects", 3GPP TR 43.030 version 9.0.0 Release 9. 